\title{
O.S.P.
}

L'orientation scolaire et professionnelle

$33 / 3$ | 2004

Varia

\section{F. Hartog. Régimes d'historicité. Présentisme et expérience du temps}

Paris : Le Seuil, 2003

Bertrand Lessault

\section{(2) OpenEdition}

Journals

Édition électronique

URL : http://journals.openedition.org/osp/752

DOI : $10.4000 /$ osp.752

ISSN : 2104-3795

Éditeur

Institut national d'étude du travail et d'orientation professionnelle (INETOP)

Édition imprimée

Date de publication : 15 septembre 2004

Pagination : 479-483

ISSN : 0249-6739

Référence électronique

Bertrand Lessault, «F. Hartog. Régimes d'historicité. Présentisme et expérience du temps », L'orientation scolaire et professionnelle [En ligne], 33/3 | 2004, mis en ligne le 28 septembre 2009, consulté le 21 septembre 2020. URL : http://journals.openedition.org/osp/752 ; DOl : https://doi.org/10.4000/osp 752

Ce document a été généré automatiquement le 21 septembre 2020.

(c) Tous droits réservés 


\section{F. Hartog. Régimes d'historicité. Présentisme et expérience du temps}

Paris : Le Seuil, 2003

Bertrand Lessault

\section{RÉFÉRENCE}

Paris : Le Seuil

1 Dans «Régimes d'historicité. Présentisme et expérience du temps », François Hartog, historien et directeur à l'école des hautes études en sciences sociales à Paris, ne se propose pas d'expliquer les phénomènes historiques contemporains. Il les saisit de biais, et s'interroge sur les temporalités qui les structurent ou les ordonnent. L'auteur à observé la montée rapide de la catégorie du présent, jusqu'à ce que s'impose l'évidence d'un présent omniprésent, ce qu'il nomme "présentisme ». La notion de "régime d'historicité » peut s'entendre de deux façons :

- Dans une acception restreinte : comment une société traite de son passé ?

- Dans une acception large : le « régime d'historicité » servirait à désigner la modalité de conscience de soi d'une communauté humaine.

\section{Ordre du temps 1}

2 La notion de " régime d'historicité » est conçue comme un outil heuristique, aidant à mieux appréhender, non le temps, mais principalement les moments de crise du temps, ici et là, quand les articulations du passé, du présent et du futur viennent justement perdre de leur évidence. La réflexion part de Lévy-Strauss qui postule que le degré d'historicité des sociétés est le même, mais que l'image subjective qu'elles se font d'elles-mêmes, la manière dont elles ressentent cette historicité, diffère. Autrement dit, les manières de vivre et de penser cette historicité, les façons d'articuler passé, présent, futur, varient. 
3 L'auteur cite Marshall Sahlins, ethnologue et historien de Fidji, qui déploie une série de micro-analyses visant à caractériser l'histoire de ces îles ou, mieux encore, leur mode d'être historique : leur façon de vivre, de faire, de raconter leur histoire. Les Maoris pensent que "le futur est derrière eux ». Habiles mythologues, ils arrivent toujours à sélectionner une tradition qui leur permet de donner une forme et une expression à leurs "intérêts» concrets du moment. L'événement n'en est pas un, au sens de l'histoire européenne moderne, qui repose sur l'idée que l'événement est unique. Ici, au contraire, l'événement est immédiatement perçu «selon l'ordre reçu de la structure, comme identique à l'événement originel ». Sahlins jette un vif éclairage sur ces phénomènes. Il montre les écarts respectifs des logiques d'interprétation, les quiproquos et les décalages, bref : la manière dont chacun constitue l'événement en fonction de ses expériences et de ses attentes.

Dans l'exemple de l'Odyssée, récit d'Homère, le mouvement même du projet narratif chanter le retour d'Ulysse - se déploie entre le passé des départs et le futur d'un retour, toujours escompté et sans cesse reporté. L'Odyssée vient après l'Iliade, elle en est l'épilogue. Dans l'Iliade, Troie n'est pas encore prise, nous sommes avant, dans l'attente. Le futur de l'Iliade apparaît comme un passé pertinent pour l'Odyssée. Dès que s'ouvre l'Odyssée, nous sommes dans l'après, dans la mémoire de l'événement et dans le souvenir des deuils et des souffrances endurées. La guerre de Troie est l'événement axial par rapport auquel l'Odyssée qui en raconte plusieurs épisodes est déjà en position d'histoire. Ulysse, chez les Phéaciens, face à l'aède (poète conteur), écoute le récit de ses propres actions : ce qui a été pur événement devient maintenant histoire car nous rencontrons la première mise en récit de l'événement. Ulysse est le seul à savoir d'expérience que ce récit est à la fois son histoire et l'Histoire. À travers la narration de ses voyages, les épisodes se relient les uns aux autres, une chronologie narrative s'instaure, une scène au présent/passé est suivie par une autre. Insensiblement le récit contraint le temps. L'ordre du récit devient un ordre du temps. L'Ulysse, parti de Troie, finira par rejoindre celui qui a fait naufrage chez les Phéaciens pour ensuite revenir à Ithaque.

5 F. Hartog ne peut prétendre faire l'essai de la notion de « régime d'historicité » dans le temps moderne et jusqu'à notre présent, sans lui faire traverser l'ordre chrétien du temps. Saint Augustin, s'adressant à son interlocuteur divin, met en scène son interrogation : qu'est-ce donc que le temps? «Si personne ne me pose la question, je sais; si quelqu'un me pose la question et que je veuille l'expliquer, je ne sais plus ». Comment puis-je savoir et ne pas savoir? Dans le rapport au temps, ce que le christianisme a apporté en propre, c'est la brisure du temps en deux par l'événement décisif de l'incarnation : la naissance, la mort et la résurrection du Fils de Dieu fait homme. Ce qui est nouveau dans le nouveau testament, c'est la tension instaurée entre le présent et l'avenir, entre l'événement décisif par quoi tout est déjà accompli et le dénouement final qui montre que tout n'est pas encore achevé. De cette tension instauratrice découle l'ordre proprement chrétien du temps et de l'histoire comme histoire du salut dans lequel le « déjà » et « le pas encore » ne s'équilibrent pas comme les deux plateaux d'une balance. Le déjà pèse plus lourd, puisque avec lui l'histoire a basculé : à partir du point décisif, le monde est sauvé. Il s'ensuit que le présent n'abolit en aucune façon le passé. Il l'éclaire, lui donne sens comme préparation et vient l'accomplir. 
Chateaubriand a mieux compris que beaucoup de ses contemporains le nouvel ordre du temps des modernes. Il a grandi en une période de crise intense et de remises en question des rapports au temps. À travers l'analyse de «l'essai historique sur les révolutions ", publié pour la première fois en 1797 (puis en 1826), l'écrivain se montre à la charnière entre deux temps: "J'écrivais l'histoire ancienne et l'histoire moderne frappait à ma porte ». Chateaubriand met en scène le décalage entre la vie besogneuse de l'historien et le mouvement rapide de l'histoire. «L'essai historique » est d'abord un récit de voyage, voyage vers le nouveau monde sans nul doute, mais d'abord voyage intérieur. Voici une enquête historique sur le cours des révolutions anciennes et modernes qui s'ouvre par cette interrogation: «qui suis-je?». Qui suis-je, effectivement, parce que le monde où je suis né s'est effondré ? Chateaubriand veut comprendre, mais aussi prévoir, en considérant les révolutions anciennes et modernes dans leurs rapports avec la révolution française. Aussi part-il du passé pour rejoindre le présent et si possible, allant plus loin, pronostiquer l'avenir. Dans «le voyage en Amérique ", publié en 1827, 36 ans après le voyage réel, le temps est au cœur du livre. Plus encore qu'un récit de voyage au premier degré, c'est en effet une Amérique revisitée et une réflexion sur l'Amérique que propose Chateaubriand. Il esquisse une histoire de la liberté ancienne et de la liberté moderne. Il voit les États-Unis non seulement comme la terre d'invention de la liberté nouvelle, mais aussi comme le laboratoire où s'est effectué " presque sans effort » et rapidement le passage de l'une à l'autre. Le miracle de l'histoire américaine est le produit d'une accélération du temps.

\section{Ordre du temps 2}

7 L'auteur inscrit le régime d'historicité où le futur prévaut sur le présent entre deux dates symboliques: 1789 et 1989. 1789, date de la révolution française, a pu être déchiffré, notamment, comme conflit entre deux régimes d'historicité: ancien et moderne. 1989, c'est la chute du mur de Berlin, le 9 novembre. Ces deux dates marquent deux césures, deux failles dans l'ordre du temps. Un " régime d'historicité » n'est pas uniquement l'expression d'un ordre dominant du temps. Tissé de différents régimes de temporalité, il est une façon de traduire et d'ordonner des expériences du temps, des manières d'articuler passé, présent et futur et de leur donner un sens.

8 Le $\mathrm{XX}^{\mathrm{e}}$ siècle a allié futurisme et présentisme. S'il a d'abord été plus futuriste que présentiste, il a fini plus présentiste du futuriste. « Futuriste » est à entendre ici comme la domination du point de vue du futur: le progrès comme unique horizon temporel. Peu à peu, au cours du dernier siècle, l'avenir s'est mis à céder du terrain au présent, ce dernier prenant de plus en plus de place, jusqu'à sembler, depuis peu, l'occuper toute entier. On est alors entré dans un temps de prévalence du point de vue du présent : le "présentisme ». Le présent est devenu l'horizon sans futur et sans passé. Il génère, au jour le jour, le passé et le futur dont il a, jour après jour, besoin et valorise l'immédiat.

Cette analyse conduit F. Hartog à s'interroger sur la place de l'historien. Pierre Nora proposait, par exemple, en 1974, un type nouveau d'histoire. Récusant le postulat d'une coupure entre le passé et le présent, il considère qu'il revient à l'historien du présent de faire consciemment surgir le passé dans le présent. La réflexion de Nora à propos de "l'événement" suggère l'existence d'un rapport entre le statut nouveau donné à l'événement, dans une société de consommation, et une façon d'appréhender le temps. Pris dans le temps de la consommation, le temps devient lui-même un objet de 
consommation. Pour Nora, «l'accélération » du temps constitue une rupture avec le passé. La mondialisation, la démocratisation, la massification entraînement la disparition de la mémoire. Tant et si bien qu'on ne parle tant de mémoire que par ce qu'il n'y en a plus. Depuis les années 1980, on ne cesse de commémorer au nom de la trilogie : mémoire / identité / patrimoine.

En 1989, avec la chute du mur de Berlin, il y a césure dans l'ordre du temps. Le futur est toujours là, plus imprévisible que jamais. Par exemple, comment l'Allemagne se vit-elle désormais en tant que nation? Alors qu'en 1980, l'Allemagne, non encore réunifiée, se définissait comme un état "post national ", se multipliaient déjà les colloques sur l'identité allemande, bien que l'on fut alors convaincu, des deux côtés du mur, du caractère durable de la coupure en deux États. La notion de patrimoine a pris, elle aussi, de plus en plus d'importance: on passe de "l'histoire mémoire" à "l'histoire patrimoine ». Le patrimoine devient symbole d'identité, de mémoire de l'histoire. Depuis 30 ans, ce présent est obsédé de mémoire. À la confiance dans le progrès s'est substitué le souci de sauvegarder, de préserver. La destruction du mur de Berlin, suivie de sa muséification instantanée, en a été un bel exemple, avec, tout aussi immédiate, sa marchandisation : ont été aussitôt mis en vente des échantillons, dûment estampillés "Original Berlin Mauer». Du point de vue du rapport au temps, cette prolifération patrimoniale est signe d'une rupture entre un présent et un passé, du sentiment vécu de l'accélération, du basculement d'un régime de mémoire dans un autre, dont Pierre Nora a fait le point de départ de son interrogation: la rupture avec le champ d'expérience, le monde où l'on a vécu, que nos aïeuls ont connu, bascule vers un monde que nous avons perdu. Le patrimoine est un recours pour temps de crise.

11 F. Hartog développe, au long de ces pages, la notion de "présentisme » qu'il conçoit, d'abord, par opposition au «futurisme » qui aurait régné auparavant et qui aurait disparu de l'horizon européen, alors que s'ouvrait un temps désorienté et que montaient les incertitudes. Cette notion lui permet, ensuite, de confronter le présent d'aujourd'hui à ceux du passé, ceux qui ont laissé le plus de traces dans la culture européenne : le présent homérique en est un exemple. L'auteur termine son propos en soulignant les principes de responsabilité et de précaution :

- La responsabilité des contemporains endettés vis-à-vis des hommes à venir commence aujourd'hui : elle est de chaque jour pour que perdure l'humanité.

- Le principe de précaution face à la crise du progrès et aux dangers de nouvelles technologies : celles-ci sont craintes eu égard aux menaces de modifications irréversibles de l'environnement et celles suscitées par les biotechnologies.

12 État durable ou de transition, le présent s'est étendu tant en direction du futur que du passé. On parle de présent multidirectionnel ou multiple pour conjurer l'insupportable incertitude de ce qui arrive. Nous ne cessons de regarder en avant et en arrière, mais sans sortir d'un présent, un présent monstre, dont nous avons fait notre seul horizon.

Dans quelle mesure ces analyses relatives aux «modes d'historicité » permettent-elles d'éclairer les pratiques actuelles d'aide à l'orientation? Ce présent massif, envahissant, omniprésent, ne tente-t-il pas de s'y insinuer, en ce sens que la consommation du conseil en orientation pourrait se banaliser? De plus, si tenir conseil répond aux besoins immédiats comme le suggère la politique adéquationniste, alors le présentisme s'avèrerait installé.

14 Mais l'acte de s'orienter, l'acte d'orientation est plus complexe qu'il ne paraît. Au départ, avant l'entretien, le sujet attend de l'extérieur des réponses tangibles, être 
renseigné sur son propre fonctionnement et trouver une solution pour son avenir qui lui convienne.

15 C'est ensuite, au cours du conseil, que l'individu fera un travail et un retour sur lui même pour se définir, se projeter. Le conseiller l'amène à se positionner. "Pour préparer des décisions, il faut évaluer la pertinence des moyens vis-à-vis des finalités, ordonner dans le temps les étapes de l'action avec leurs buts intermédiaires. C'est par rapport à cela que des décisions stratégiques et tactiques peuvent être prises » (C. Chabrol, p. 178, O.S.P. mars 2000).

16 Les pratiques en orientation amènent à une réflexion de soi en lien avec le facteur temps. C'est pour cela que la démarche d'orientation est une démarche de construction de soi dans une dimension temporelle. La question est : «Que vais-je faire de moi, avec ce que l'on a fait de moi ?"

17 "Cette dimension temporelle de l'image de soi est centrale dans les problématiques de l'orientation où toutes les conduites sont orientées à moyen et long terme vers le futur » (Huteau et Guichard, p. 90, Psychologie de l'orientation 2000). Le Conseiller d'Orientation-Psychologue, par ses pratiques, est une sorte de garant qui instruit les consultants à se conduire comme acteur de leur devenir et à acquérir une prise de conscience pour rester critiques sur ce qu'ils peuvent recevoir.

Le présentisme est là, nous ne sommes plus ignorants de son existence. Les pratiques d'aide à l'orientation offrent dans la prise en charge individuelle un espace d'écoute et de dialogue où la personne reçue pourra faire le point sur ses envies, réfléchir à ses priorités, évaluer ses possibilités, élaborer des stratégies ou s'interroger sur le sens de ses comportements par rapport à ce régime d'historicité. 